\title{
Scientific Sessions
}




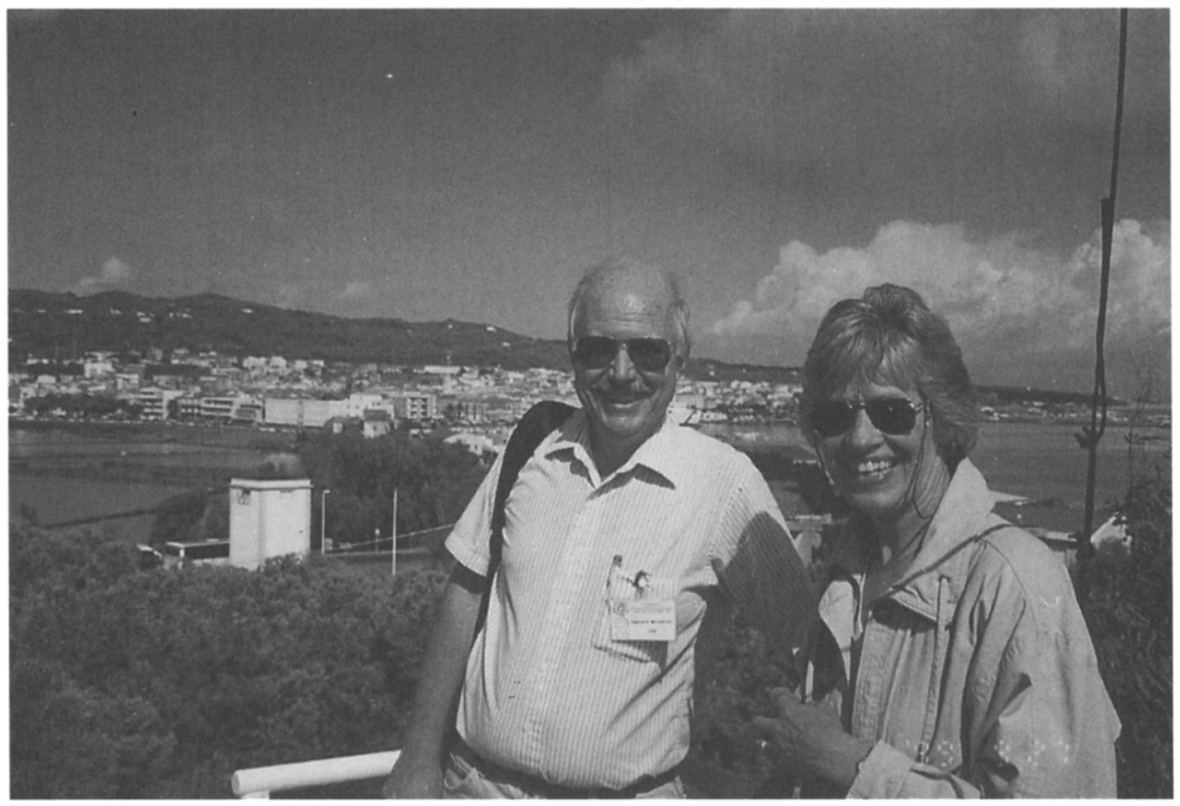

Dennis and Diane McCarthy 


\title{
Polar Motion - An Overview
}

\author{
Dennis D. McCarthy \\ U. S. Naval Observatory \\ 3450 Massachusetts Ave., NW \\ Washington DC 20392-5420, USA \\ e-mail:dmc@maia.usno.navy.mil
}

\begin{abstract}
After one hundred years of intensive study, some aspects of polar motion remain poorly understood. This motion of the Earth's axis of rotation with respect to its mantle and crust has been observed and studied since the creation of the International Latitude Service. Research has shown that the motion of the pole is quite complicated and distinguished by components over all time scales. Observationally, the secular, Chandler and annual components of polar motion are well documented, and various geophysical processes have been suggested as likely causes. Other components such as the approximately thirty-year motion, the high-frequency motion and the daily/sub-daily motion remain as subjects of extensive research.
\end{abstract}

\section{Introduction}

Polar motion is one of the components of the set of angles specifying the orientation of the Earth in an inertial reference frame. The Earth's orientation is specified commonly using five angles. These are the precession/nutation angles $\psi$ (the longitude of the ascending node of the ecliptic) and $\epsilon$ (the obliquity of the ecliptic), the rotation angle of the Earth as specified by the time difference (UT1-UTC) and the polar motion angles $\mathrm{x}$ and $\mathrm{y}$. The nutation angles provide the orientation of the Celestial Ephemeris Pole (CEP) in the Celestial Reference Frame. UT1-UTC specifies the angle through which the International Reference Meridian has turned with respect to a fiducial point on the Earth's equator, and the polar motion angles describe the position of the Celestial Ephemeris Pole with respect to the International Terrestrial Reference Frame (McCarthy 1996). While three angles would suffice to specify the Earth's orientation in an inertial frame, five angles are used in order to describe the results of the physical processes that are occurring.

The International Celestial Reference Frame (ICRF) is a set of angular coordinates that is used to realize the International Celestial Reference System (ICRS). The precession/nutation angles describe the angular orientation of the Celestial Ephemeris Pole in the ICRS. The polar motion angles $\mathrm{x}$ and $\mathrm{y}$ are defined in the International Terrestrial Reference System (ITRS) realized by the International Terrestrial Reference Frame (ITRF). The latter is a set of precisely defined station coordinates including site positions and motions. The angles $\mathrm{x}$ 


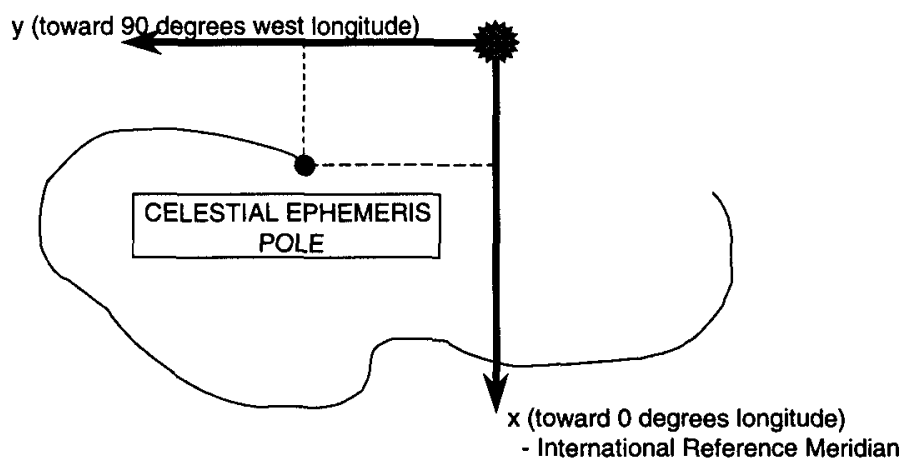

Figure 1. Polar motion angles $\mathrm{x}$ and $\mathrm{y}$.

and $y$ are components of the orientation of the CEP in the ITRF, the $x$-axis being directed along the International Reference Meridian and $\mathrm{Y}$ along the meridian $90^{\circ}$ West longitude. Figure 1 shows the way in which the angles are defined and how the rotation angle UT1-UTC is defined with respect to a fiducal point defined in the ICRS.

Mathematically the relationship between coordinates in the ITRF and those in the ICRF can be given as

$$
\left[\begin{array}{l}
X \\
Y \\
Z
\end{array}\right]_{I C R F}=[P N(t)][R(t)][W(t)]\left[\begin{array}{l}
X \\
Y \\
Z
\end{array}\right]_{I T R F},
$$

where $[P N(t)]$ is the precession/nutation rotation matrix, $[R(t)]$ is the rotation angle matrix, and $[W(t)]$ is the polar motion matrix,

$$
[W(t)]=\left[\begin{array}{ccc}
1 & 0 & -x \\
0 & 1 & y \\
x & -y & 1
\end{array}\right] .
$$

The polar motion angles are subject to variations as described by the equations

$$
\frac{d}{d t} \vec{H}+\vec{\varpi} \times \vec{H}=\vec{L},
$$

$\vec{H}=$ angular momentum $=I \vec{\varpi}+\vec{h}$,

$I=$ inertia tensor,

$\vec{\varpi}=$ Earth's rotation vector, 
$\vec{h}=$ relative angular momentum, and

$L=$ torque.

\section{Observations}

Astronomical observations of polar motion have been carried out for over one hundred years. A variety of instruments and techniques have been employed during that time. The accuracy of these observations has continued to improve as technology has advanced

\subsection{Observational Instrumentation}

Optical observations of stars were the first to be used to establish the existence of polar motion and to continue its monitoring. Transit circles and zenith telescopes were the primary optical instruments, but both visual and photographic zenith telescopes were employed until the late 1980 s as a significant source of polar motion information. Doppler observations of navigational satellites were used to improve determinations of polar motion during the 1970s and 80s. Modern techniques came into existence in the 1970s when laser ranging to artificial Earth satellites and very long baseline radio interferometry (VLBI) began to make important contributions to our observational knowledge of polar motion. Currently, polar motion information is obtained principally from observations of the satellites of the Global Positioning System (GPS) and VLBI. Reviews of the observational techniques can be found in Lambeck (1988) and Eubanks (1993). See also Mireault et al. (1999) regarding GPS-determined polar motion from the International GPS Service.

\subsection{Sources of Observations}

The International Latitude Service (ILS) was the first source of operational estimates of polar coordinates, using instrumentation consisting only of visual zenith telescopes. As more optical instrumentation became available, the ILS was transformed into the International Polar Motion Service (IPMS) which included not only the visual zenith telescopes of the ILS, but also the visual and photographic telescope data of other international institutions. These observations have recently been reduced in the Hipparcos reference frame (Vondrák et al. 1997, Vondrák 1999). The Bureau International de l'Heure (BIH) began the routine collection of polar motion data to facilitate its mission to determine UT1. Determination of astronomical time (UT1) relied on accurate determination of polar motion, and the BIH collected and published polar motion information to provide a consistent set of UT1 and polar motion data. The International Earth Rotation Service (IERS) began operation in 1988 following the recommendation of the MERIT Working Group. Essentially this service combined the operation of the IPMS and the BIH into one organization that made optimal use of the most accurate astronomical observations available to provide the ICRS, the ITRS and the means to relate them to each other. Brief histories of these organizations can be found in Yokoyama (2000), Guinot (2000), Wilkins (2000), and Mueller (2000). Time series of polar motion are currently available from the IERS. 


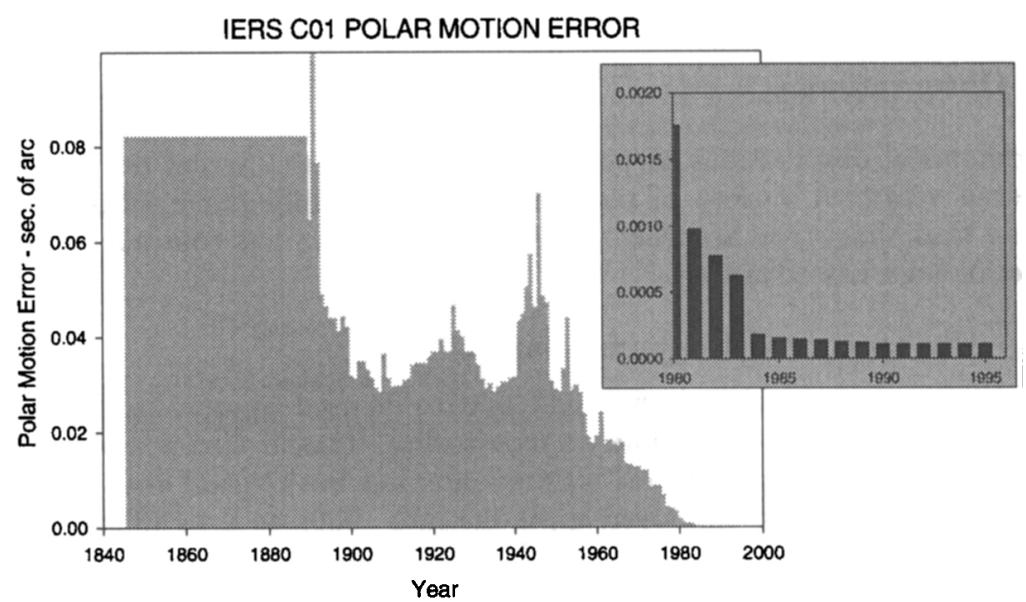

Figure 2. The improvement of the accuracy with which polar motion can be determined as a function of time. Data are taken from IERS polar motion solution IERS C01.

\subsection{Observational Accuracy}

Figure 2 shows the improvement of observational accuracy through time. Currently we expect daily estimates of polar motion accurate to \pm 0.1 milliarcseconds (mas) corresponding to a measurement of a few millimeters on the Earth's surface.

\subsection{Observational Data}

Figure 3 shows the path of the pole from 1990 to 1999, and Figure 4 shows the $\mathrm{x}$ and $\mathrm{y}$ components of the polar motion as a function of time. Obvious from these plots are the facts that the polar motion has a linear trend (as seen in the linear increase in $y$ with time) and a series of cyclical variations. Figure 5 shows a schematic power spectrum of these periodic variations.

\subsection{Analysis of Observational Data}

Past analyses of observational data make it clear that polar motion can be separated into components corresponding with the periodic frequency of the motion. These components are the secular motion, the decadal motion, the Chandler and annual motions, the high-frequency motion and the diurnal/subdiurnal motions. Reviews of the components of polar motion can be found in Munk and MacDonald (1960), Rochester (1973), Lambeck (1980), and Eubanks (1993).

Secular Motion Secular polar motion is the apparently aperiodic motion of the pole toward the direction of approximately $75^{\circ}$ west longitude. Figure 6 displays 


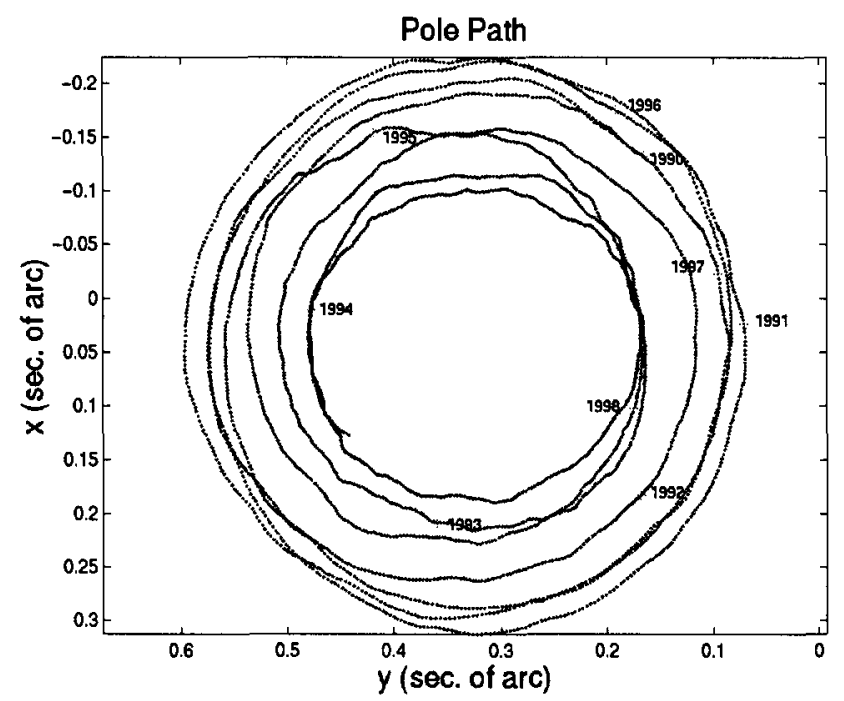

Figure 3. Path of the Celestial Ephemeris Pole from 1990 to 1999. Data are from polar motion solutions of the National Earth Orientation Service (NEOS).
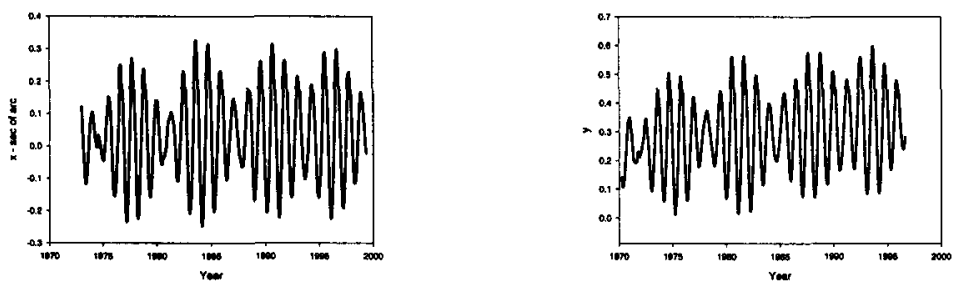

Figure 4. Components of polar motion as a function of time. 


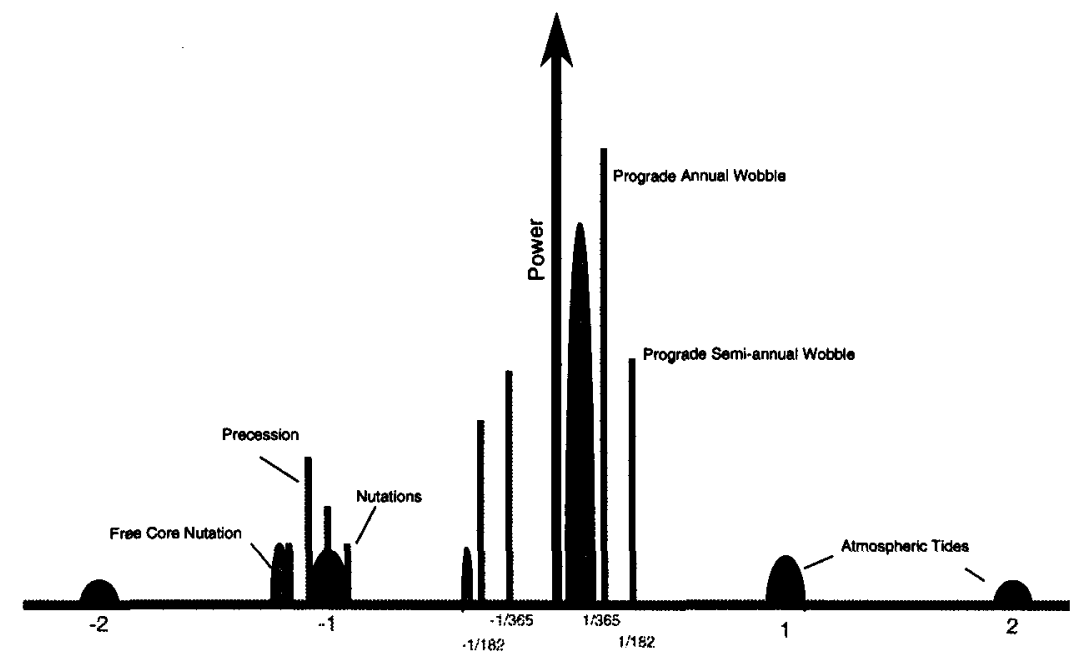

Frequency in the Terrestrial Reference Frame (cycles per day)

Figure 5. Schematic polar motion spectrum.

the linear fit to the $\mathrm{x}$ and $\mathrm{y}$ components of polar motion that is interpreted as a secular polar motion. For many years the reality of secular polar motion was in doubt because of questions regarding the possible systematic errors in the analysis of the astronomical observations. Recent observations using modern totally independent techniques, however, corroborate the past determinations proving that this motion is real.

The cause of secular polar motion is most likely the effects of post-glacial rebound. Other possibilities that have been raised include melting of glaciers and changes in continental water storage. The parameters of this component of polar motion are valuable sources of information used to calibrate and check viscoelastic rebound models (Johnston and Lambeck 1999, Mitrovica and Milne 1998).

Decadal Motion Decadal polar motion refers to the quasi-periodic motions of the pole with periods estimated to be between ten and forty years. The motions with amplitudes of approximately 20 mas are apparently real as they have been detected with both optical and independent modern techniques. Figure 7 displays this component of polar motion (Greiner-Mai 1997, Molodensky and Groten 1998, Pan 1999).

Chandler and Annual Motions The Chandler and annual components of polar motion (see Figure 8) dominate the power spectrum of the observations (see Figure 9). We know that the Chandler motion is periodic with a period of 


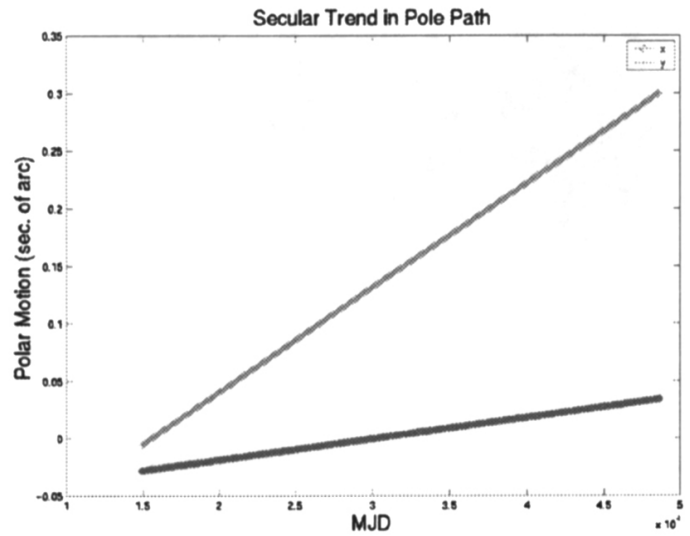

Figure 6. Linear fit to the $\mathrm{x}$ and $\mathrm{y}$ polar motion components of polar motion as a function of time. Data are taken from NEOS polar motion solutions.

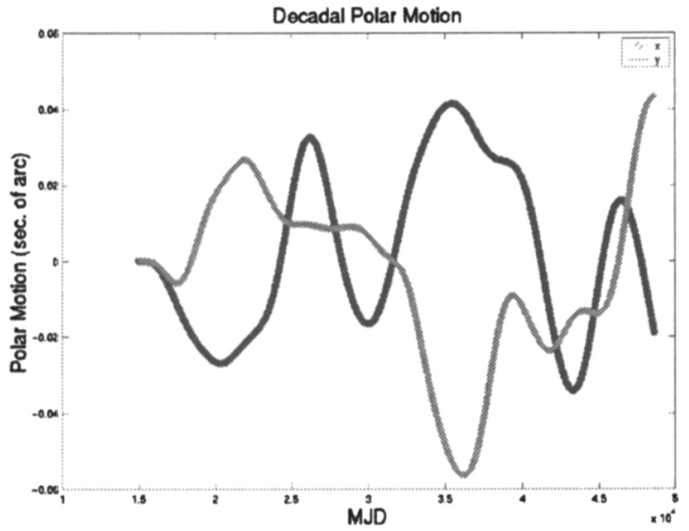

Figure 7. Components of decadal polar motion. 


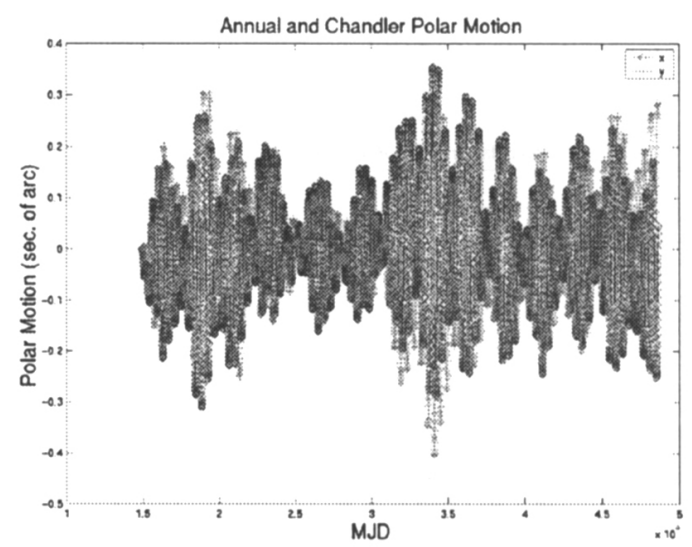

Figure 8. The Chandler and annual polar motion.

approximately 433 days. We also know that the amplitude of the motion is variable but that it is of the order of about 150 mas. The motion has a $Q$ value of about 170 implying a damping time of about 64 years. The possible excitation of this motion remains mysterious (Vicente and Wilson 1997). Possible causes include atmospheric, ground water, and seismic excitation as well as core mantle torque.

Annual or seasonal polar motion is a stable prograde motion with an amplitude of approximately 90 mas. Causes are apparently atmospheric, oceanic, and ground water excitations (deViron et al. 1999, Dickman 1998, Dickey et al. 1999, Gu 1996, Höpfner 1996, Sidorenkov 1997, Kołaczek et al. 2000).

High-frequency Motions The high-frequency portion of the polar motion spectrum contains a series of quasi-periodic motions with amplitudes of the order of one mas. (see Figure 10 for a display of the high-frequency motions.) These motions are poorly understood but causes are assumed to be atmospheric and oceanic (Brosche et al. 1997, Ponte et al. 1998, Gross et al. 1998, Yu and Zheng 1998, Nastula and Salstein 1999).

Diurnal/Sub-diurnal Motions Recent high-precision observations have confirmed the existence of diurnal and sub-diurnal polar motion (Figures 11 and 12). These motions are caused by ocean tides and reasonably modellable with amplitudes of the order of one mas (Brosche and Schuh 1998).

\subsection{Remaining Questions}

While many aspects of polar motion are becoming better understood, both in terms of observations and theory, some questions remain. Among these questions are the causes of the long-period (decadal) polar motion and the variations in the amplitude of the Chandler motion. The total cause or causes of the highfrequency, subseasonal motions also remains enigmatic. All of these features of 

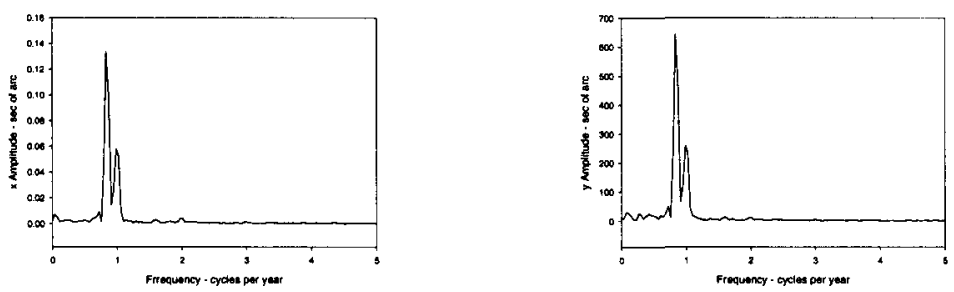

Figure 9. Power spectrum of polar motion observations showing the dominance of the Chandler and annual periodic variations.

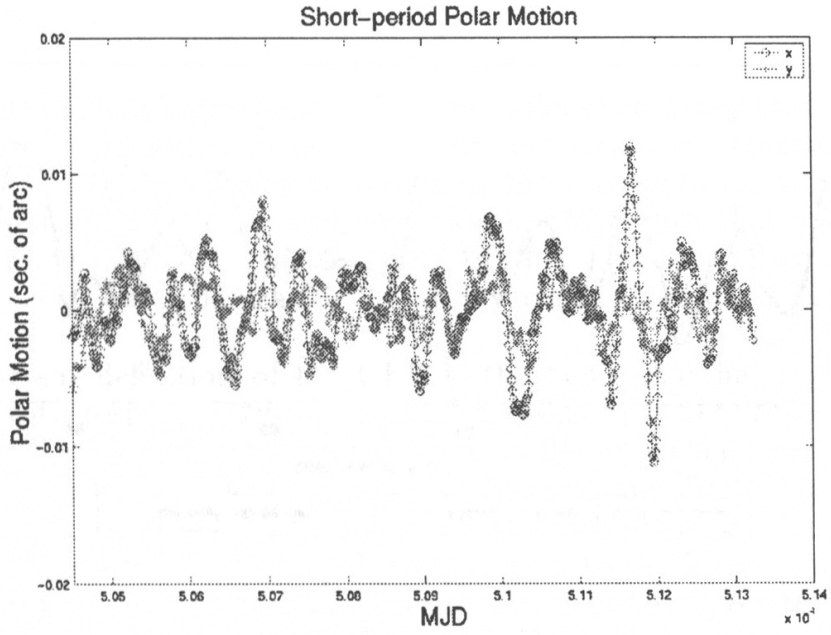

Figure 10. High-frequency polar motion. 


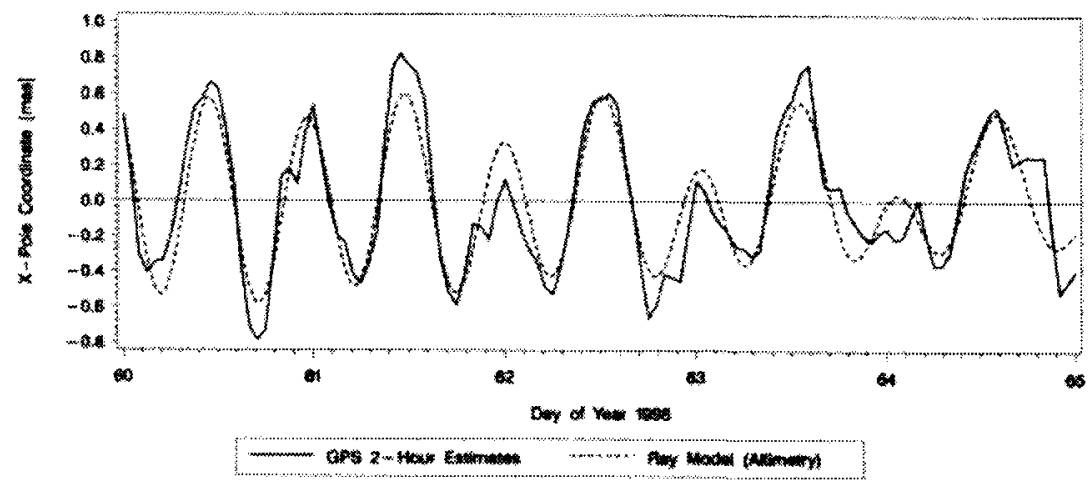

Figure 11. Diurnal/subdiurnal variation in the $\mathrm{x}$ component of polar motion.

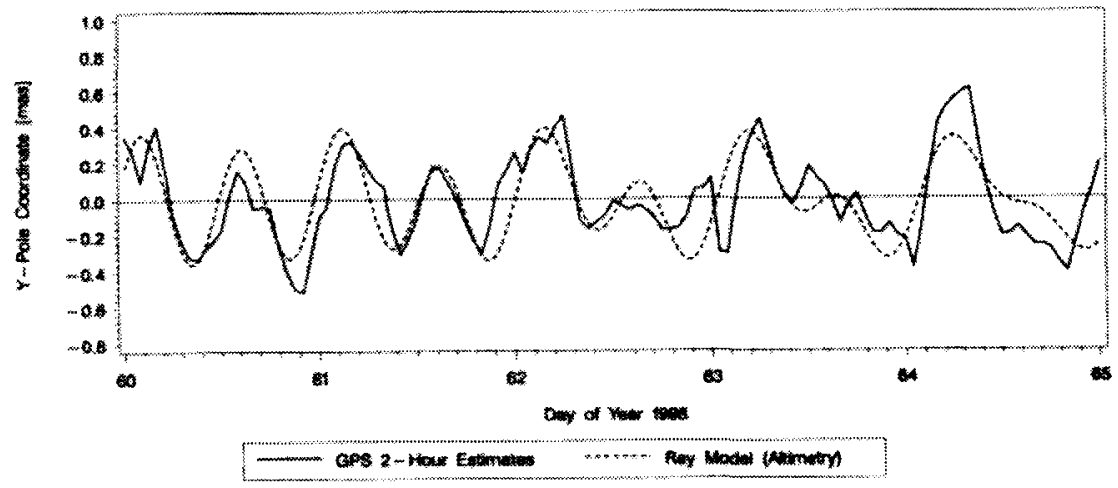

Figure 12. Diurnal/subdiurnal variation in the $y$ component of polar motion. 


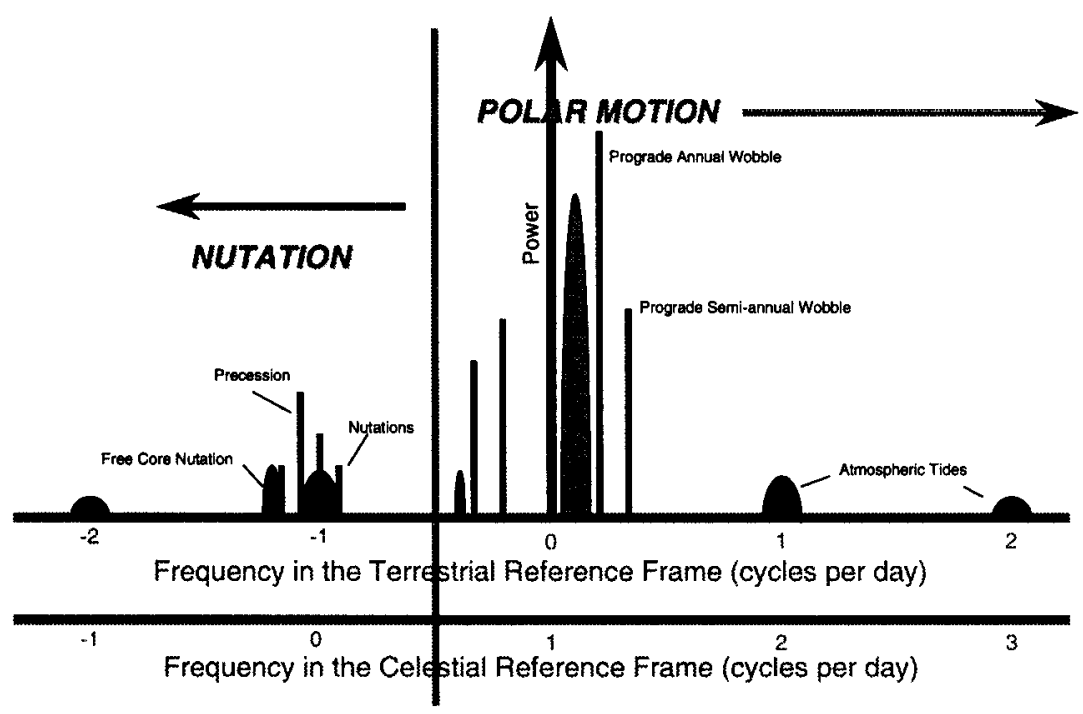

Figure 13. Possible division of the polar motion and precession/nutation spectrum.

polar motion are well-documented observationally but remain to be explained totally physically.

\section{Issues}

Ironically, the modern improvements in observational accuracy have raised issues with the current definition of polar motion and nutation. Questions are also raised about the optimum way to represent the total rotation from terrestrial to celestial reference systems (and vice versa). Along with that question is the concern over how often observational data will be required to provide users with accurate information to determine the proper transformations (Capitaine et al. 1999.

The current definition of the CEP is the "pole that has no nearly diurnal motion with respect to the space-fixed coordinate system or an Earth-fixed coordinate system," or the "pole of the axis of figure for the mean surface of a model of the Earth in which the free motion has zero amplitude." In practice the motion of this pole in the celestial reference system is realized through VLBI observations of the "offsets" of the pole with respect to the International Celestial Reference System. Analyses of VLBI observations however, obtain these offsets by assuming that diurnal variations can be assumed to be precession/nutation. It is now apparent that there is real polar motion at these same frequencies. This situation blurs the distinction between polar motion and precession/nutation.

Using currently endorsed definitions, nutation consists of long-period terms in the celestial frame and polar motion consists of long period terms in the 
Table 1. Separation of polar motion from precession/nutation by cause.

\begin{tabular}{|c|l|c|c|c|c|c|c|c|c|}
\hline & & \multicolumn{3}{|c|}{ Short Period } & \multicolumn{5}{c|}{ LongPeriod } \\
\hline & & Predictable & \multicolumn{2}{|c|}{ Unpredictable } & \multicolumn{2}{c|}{ Predictable } & \multicolumn{2}{c|}{ Unpredictable } \\
\hline & & CRS & TRS & CRS & TRS & CRS & TRS & CRS & TRS \\
\hline 1 & Polar Motion & & & & & & X & & X \\
\hline & Nutation & & & & & X & & X & \\
\hline & & & & & & & & & \\
\hline 2 & Polar Motion & & $\mathrm{X}$ & $\mathrm{X}$ & $\mathrm{X}$ & & $\mathrm{X}$ & $\mathrm{X}$ & $\mathrm{X}$ \\
\hline & Nutation & $\mathrm{X}$ & & & & $\mathrm{X}$ & & & \\
\hline & & & & & & & & & \\
\hline 3 & Polar Motion & $\mathrm{X}$ & $\mathrm{X}$ & $\mathrm{X}$ & $\mathrm{X}$ & & $\mathrm{X}$ & $\mathrm{X}$ & $\mathrm{X}$ \\
\hline & Nutation & & & & & $\mathrm{X}$ & & & \\
\hline
\end{tabular}

terrestrial frame. The question arises over the fact that there is no satisfactory definition of the short-period effects.

Two general suggestions are (1) to separate the effects by frequency, and (2) to separate the effects by cause. In the first suggestion, it has been proposed to separate the effects by splitting the frequency spectrum into a polar motion portion and a nutation portion. (See Figure 13.)

The alternate suggestion is to divide the phenomena into areas that are predictable and those that are not. At least three possibilities are shown in Table 1.

\section{Conclusion}

During the past one hundred years, the accuracy with which observations of polar motion have been made has improved three orders of magnitude. This has made it possible to understand the geophysical causes for polar motion. Many aspects of polar motion remain enigmatic.

\section{References}

Brosche, P., Wünsch, J., Maier-Reimer, E., Segschneider, J., and Sündermann, J., 1997, "The axial angular momentum of the general circulation of the oceans," Astron. Nachr., 318, pp. 193-199.

Brosche, P. and Schuh, H., 1998, "Tides and Earth Rotation," Surveys in Geophysics, 19, pp. 417-430.

Capitaine, N., Guinot, B., and McCarthy, D. D., "The Celestial Ephemeris Origin and the definition of UT1 in the International Conventional Reference Frame, Astron. Astrophys., in press. 
deViron, O., Bizouard, C., Salstein, D., and Dehant, V., 1999, "Atmospheric torque on the Earth rotation and comparison with atmospheric angular momentum variations," J. Geophys. Res., 104, pp. 4861-4875.

Dickman, S. R., 1998, "Determination of oceanic dynamic barometer corrections to atmospheric excitation of Earth rotation," J. Geophys. Res., 103, pp. 1127-15143.

Eubanks, T. M., 1993, "Variations in the Orientation of the Earth," in Contributions of Space Geodesy to Geodynamics: Earth Dynamics, American Geophysical Union, Washington, DC.

Dickey, O., Gegout, P., and Marcus, S. L., 1999, "Earth-Atmosphere Exchange and ENSO: The Rotational Signature of the 1997-1998 Event," Geophys. Res. Lett., 26, pp. 2477-2480.

Greiner-Mai, H., 1997, "Possible relations between the rotational axis of the inner Earth's core and the magnetic dipole axis," Astron. Nachr., 318, pp. $63-74$.

Gross, R. S., Chao, B. F., and Desai, S. D., 1998, "Effect of Long-Period Ocean Tides on the Earth's Polar Motion," Progress in Oceanography, 40, pp. 385-397.

Gu, Z., 1996, "The study of excitation of the earthquake to Earth's rotation," Earth, Moon, and Planets, 74, pp. 35-47.

Höpfner, J., 1996, "Polar motion at seasonal frequencies," J. Geodyn., 22, pp. 51-61.

Johnston, P. and Lambeck, K., 1999, "Postglacial rebound and sea level contributions to changes in the geoid and the Earth's rotation axis," Geophys. J. Int., 136, pp. 537-558.

Kołaczek, B., Nuzhdina, M., Nastula, J., and Kosek, W., 2000, "El Niño impact on atmospheric polar motion excitation," J. Geophys. Res., 105, pp. 3081-3087.

Lambeck, K., 1980, The Earth's variable rotation, Cambridge University Press, Cambridge, 449 pp.

McCarthy, D. D., 1996, IERS Conventions, IERS Technical Note, 21, Observatoire de Paris, Paris.

Mireault, Y., Kouba, J., and Ray, J., 1999, 'IGS Earth rotation parameters,' GPS Solutions, 3, pp. 59-72.

Mitrovica, J. X. and Milne, G. A., 1999, "Glaciation-induced perturbations in the Earth's rotation: A new appraisal," J. Geophys. Res., 103, pp. 9851005.

Molodensky, S. M. and Groten, E., 1998, "On the dynamical effects of a heterogeneous and compressible liquid core in the theory of Chandler wobble," Geophys. J. Int., 135, pp. 723-726.

Munk, W. H. and MacDonald, G. J. F., 1960, The Rotation of the Earth, a Geophysical Discussion, Cambridge University Press, Cambridge, 323 pp.

Nastula, J. and Ponte, R. M., 1999, "Further evidence for oceanic excitation of polar motion," Geophys. J. Int., 139, pp. 123-130. 
Pan, C., 1999, "Angular momentum perturbation, polar motion excitation and axial near-symmetry," Geophys. J. Int., 136, pp. 139-148.

Ponte, R.. M. Stammer, D., and Marshall, J., 1998, "Oceanic signals in observed motions of the Earth's pole of rotation," Nature, 91, pp. 476-479.

Rochester, M., 1973, "The Earth's Rotation," EOS, Transactions American Geophysical Union, 54, pp. 769-781.

Sidorenkov, N. S., 1997, "The Effect of El-Niño Oscillation on the Excitation of the Chandler Motion of the Earth Pole," Astronomicheskii Zhurnal, 74, pp. 792-795.

Vicente, R. O., and Wilson, C. R., 1997, "On the Variability of the Chandler Frequency," J. Geophys. Res., 102, pp. 20439-20445.

Vondrák, J., Ron, C., and Pesek, I., 1997, "Earth rotation in the Hipparcos reference frame," Cel. Mech. and Dynam. Astron., 66, pp. 115-122.

Vondrák, J., 1999, "Earth Rotation Parameters 1899.7-1992.0 after Reanalysis within the Hipparcos Frame," Surv. Geophys., 20, pp. 165-195.

$\mathrm{Yu}$, N. H. and Zheng, D. W., 1998, "Oceanic angular momentum and its excitation to the Earth rotation variations," Progress in Astronomy, 16, pp. $35-40$. 